\title{
Correction to: Involvement of Midbrain Dopamine Neuron Activity in Negative Reinforcement Learning in Mice
}

\author{
Zhijun Diao ${ }^{1} \cdot$ Li Yao $^{1,4}$. Qiangqiang Cheng ${ }^{2} \cdot$ Meilin $\mathrm{Wu}^{1} \cdot$ Yuanyuan $\mathrm{Di}^{1} \cdot$ Zhaoqiang Qian $^{1} \cdot$ Chunling Wei $^{1}$. \\ Yingxun Liu ${ }^{1} \cdot$ Yingfang Tian $^{2} \cdot$ Wei Ren ${ }^{1,3}$
}

Published online: 21 October 2021

๑) Springer Science+Business Media, LLC, part of Springer Nature 2021

\section{Correction to: Molecular Neurobiology https://doi.org/10.1007/s12035-021-02515-6}

The original version of this article unfortunately missed to include the explanation of co-first author contribution. The authors, Zhijun Diao and Li Yao, contribute equally to this paper. The sentence "these authors contribute equally" should be added in this paper.

The affiliations of some authors marked incorrect. The affiliation of $\mathrm{Li}$ Yao is incorrect. It should be "Faculty of Table Tennis, Badminton and Tennis, Chengdu Sport University, Chengdu, 610041,Sichuan, China" not "College of Life Sciences, Shaanxi Normal University, Xi'an, 710119, Shaanxi, China". The affiliation of Qiangqiang Cheng and Yingfang Tian is incorrect. It should be "College of Life Sciences, Shaanxi Normal University, Xi' an, 710119, Shaanxi,
China" not "College of Psychology, Shaanxi Normal University, Xi'an, 710062, Shaanxi, China". The affiliation of Wei Ren is incorrect. It should be "College of Psychology, Shaanxi Normal University, Xi'an, 710062, Shaanxi, China" not "Faculty of Table Tennis, Badminton and Tennis, Chengdu Sport University, Chengdu, 610041, Sichuan, China".

With this, the original article was corrected.

Publisher's Note Springer Nature remains neutral with regard to jurisdictional claims in published maps and institutional affiliations.

Yingfang Tian

yingfang_tian@snnu.edu.cn

$\triangle$ Wei Ren

renwei@snnu.edu.cn

1 Key Laboratory of Modern Teaching Technology, Ministry of Education, Shaanxi Normal University, Xi'an 710062, Shaanxi, China

2 College of Life Sciences, Shaanxi Normal University, Xi'an 710119, Shaanxi, China

3 College of Psychology, Shaanxi Normal University, Xi'an 710062, Shaanxi, China

4 Faculty of Table Tennis, Badminton and Tennis, Chengdu Sport University, Chengdu 610041, Sichuan, China 